\title{
Inhibition of the Vapor-Mediated Phase Transition of the High Temperature Form of Pyrazinamide
}

\author{
M. M. H. Smets, ${ }^{\dagger}$ G. Baaklini, ${ }^{\dagger}$ A. Tijink, ${ }^{\dagger}$ L. Sweers, ${ }^{\dagger}$ C. H. F. Vossen, ${ }^{\dagger}$ C. Brandel, ${ }^{\ddagger}$ H. Meekes, ${ }^{\dagger}$ \\ H. M. Cuppen, ${ }^{* \dagger}$ and G. Coquerel ${ }^{*}+$
}

${ }^{\dagger}$ Institute for Molecules and Materials, Radboud University, Heyendaalseweg 135, 6525 AJ, Nijmegen, The Netherlands

${ }^{\ddagger}$ Normandie Université, Laboratoire SMS-EA3233, Université de Rouen, F-76821, Mont-Saint-Aignan, France

\section{Supporting Information}

ABSTRACT: Tailor-made additives can prove an effective method to prolong the lifetime of metastable forms of pharmaceutical compounds by surface stabilization. Pyrazinamide (PZA) is a pharmaceutical compound with four polymorphic forms. The high temperature $\gamma$ form, which can be produced by spray drying or sublimation growth, is metastable at room temperature and transforms within days when produced by spray drying, and within several months up to years for single crystals produced by sublimation. However, when PZA is cospray dried with 1,3dimethylurea (DMU), it has been reported to remain in its $\gamma$ form for several years. Scanning electron microscopy (SEM) images reveal that the phase transition from $\gamma$-PZA to the low temperature forms

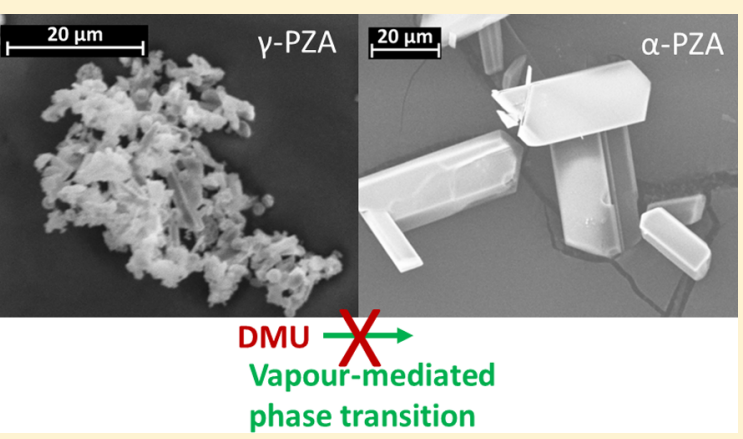
involves a vapor-mediated recrystallization, while the reverse phase transition upon heating is a nucleation-and-growth solidsolid phase transition. The lifetime-extending effect of DMU on spray-dried PZA has been investigated in more detail and compared with high-energy ball milling of sublimation-grown $\gamma$-PZA crystals. Co-ball milling of PZA and DMU is found to extend the lifetime of the high temperature form of PZA to a few months, while separate ball milling leads to an extension of merely a few weeks. DMU acts as an additive that most likely stabilizes the surface of $\gamma$-PZA, which would reduce the vapor pressure of PZA, thereby reducing the transition rate. Alternatively, DMU could prevent nucleation of low temperature forms of PZA.

\section{INTRODUCTION}

Polymorphism is a common phenomenon in molecular crystals. ${ }^{1}$ In the pharmaceutical industry, the control of the polymorphic form is a very important issue, since different polymorphic forms can have distinct physicochemical properties such as solubility, dissolution rate, and morphology. ${ }^{2-4}$ Generally, the stable polymorph is preferred for production of active pharmaceutical ingredients (APIs) to ensure reproducible bioavailability, also after prolonged storage under various conditions. However, the stable form is usually less soluble than a metastable form, and in some cases the solubility or dissolution rate is so low that the therapeutic dose cannot be achieved. $^{5-7}$ Moreover, the morphology and other physicochemical properties of a polymorphic form can be disadvantageous for the manufacturing process; e.g., needle-shaped crystals can clog filters. Therefore, it is sometimes preferred to use a metastable form or even an amorphous form for the production of an API, but the risk of polymorphic transitions for the former and crystallization for the latter must then be carefully mitigated.

Tailor-made additives can be used for polymorph selection, by inhibiting the nucleation or crystal growth of specific polymorphic forms, allowing other forms to crystallize.,8-14 However, the challenge is to ensure a prolonged lifetime of metastable forms to prevent undesired solid-state phase transitions. ${ }^{7}$ The lifetime is determined by the kinetic barrier between the metastable form and other more stable forms of the API. Solid-state phase transitions usually start at specific defects, often on the surface of a crystallite. ${ }^{15}$ Therefore, surface stabilization by tailor-made additives might prove an effective method to increase the lifetime of a metastable polymorph. Very promising effects in this direction have been observed for the pharmaceutical compound pyrazinamide (PZA), where the high temperature form can be prevented from transforming at room temperature by the addition of 1,3-dimethylurea (DMU). ${ }^{16}$ The molecular structures of both compounds are shown in Figure 1.

Pyrazinamide $\left(\mathrm{C}_{5} \mathrm{H}_{5} \mathrm{~N}_{3} \mathrm{O}\right.$, pyrazinecarboxamide, PZA $)$ is an antibiotic against tuberculosis and has four known anhydrous polymorphic forms. ${ }^{17}$ The unit cell parameters determined at temperatures of $90-100 \mathrm{~K}$ are shown in Table 1 . The $\delta$ form is the stable form below $298 \mathrm{~K}$, according to studies in solution, ${ }^{18}$ the $\alpha$ form between 298 and $418 \mathrm{~K}$, the $\gamma$ form above $418 \mathrm{~K}$, and the $\beta$ form is probably a metastable form at all

Received: November 6, 2017

Revised: December 11, 2017

Published: December 28, 2017 
<smiles>NC(=O)c1cnccn1</smiles>

(a) Pyrazinamide<smiles>CNC(=O)NC</smiles>

(b) 1,3-Dimethylurea
Figure 1. Molecular structure of (a) pyrazinamide and (b) 1,3dimethylurea.

Table 1. Spacegroup and Unit Cell Parameters for the Four Polymorphic Forms of Pyrazinamide ${ }^{18,20-22}$

\begin{tabular}{lllll}
\multicolumn{1}{r}{ polymorph } & \multicolumn{1}{c}{ Form $\delta$} & \multicolumn{1}{c}{ Form $\alpha$} & \multicolumn{1}{c}{ Form $\beta$} & \multicolumn{1}{c}{ Form $\gamma$} \\
\hline Refcode CSD & PYRZIN16 & PYRZIN22 & PYRZIN23 & PYRZIN19 \\
space group & $P \overline{1}$ & $P 2_{1} / n$ & $P 2_{1} / c$ & $P c$ \\
$\begin{array}{l}\text { temperature } \\
\quad(\mathrm{K})\end{array}$ & 100 & 100 & 90 & 100 \\
$a(\AA)$ & & & & \\
$b(\AA)$ & 5.119 & 3.617 & 14.340 & 7.176 \\
$c(\AA)$ & 5.705 & 6.741 & 3.621 & 3.651 \\
$\alpha(\circ)$ & 9.857 & 22.463 & 10.613 & 10.663 \\
$\beta(\circ)$ & 97.46 & & & \\
$\gamma(\circ)$ & 98.17 & 92.39 & 101.04 & 106.34 \\
$V\left(\AA^{3}\right)$ & 106.47 & & & \\
$V$ per molecule & 268.8 & 547.3 & 540.9 & 268.1 \\
$\quad 134.4$ & 136.8 & 135.2 & 134.0 \\
$\left.Z / \AA^{3}\right)$ & & & & \\
$R$-factor $(\%)$ & 3.72 & 1.6 & 1.53 & 3.92 \\
\end{tabular}

temperatures. ${ }^{17}$ PZA is commercialized in the $\alpha$ form. According to Castro et al., the solid-state $\delta \rightarrow \alpha$ phase transition is observed at $395 \mathrm{~K}^{17}$ The four polymorphic forms can be crystallized from different solvents, or at different temperatures, but the $\alpha, \beta$, and $\delta$ forms can also be formed concomitantly ${ }^{19}$ at room temperature. The high temperature $\gamma$ form can be obtained by spray drying or sublimation growth. In the energy-temperature phase diagram (Figure 2) the stability

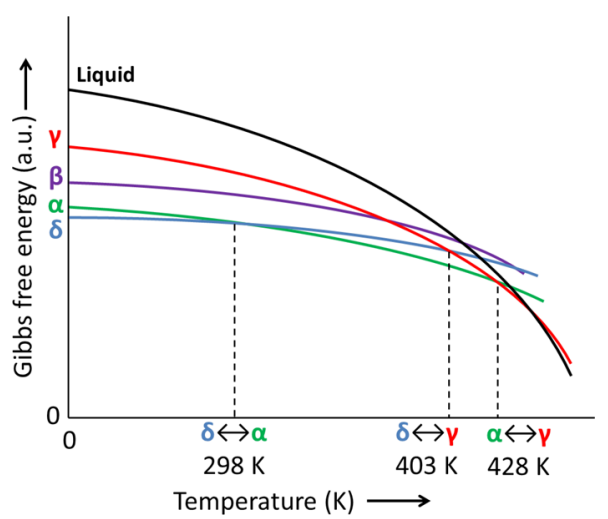

Figure 2. Schematic energy-temperature diagram of pyrazinamide, showing the stability relations between the four polymorphic forms and the liquid phase, based on the transition temperatures in ref 17 .

relation between the polymorphic forms and the liquid phase are shown schematically. However, the thermodynamic transition temperatures are not exactly known. The melting point of PZA is $461 \mathrm{~K}$ according to differential scanning calorimetry (DSC) measurements. ${ }^{17}$

The $\alpha, \beta$, and $\delta$ polymorphic forms all consist of head-tohead dimers, although the stacking of the dimers is different in each polymorphic form. ${ }^{17}$ The typical morphologies of the four polymorphic forms grown from solution or sublimation are shown in ref 17 . In contrast, the $\gamma$ form is the only noncentrosymmetric variety and consists of head-to-tail chains, but it has a spatial arrangement similar to the $\beta$ form. Therefore, the transformation from the $\gamma$ form to the other polymorphic forms and vice versa will entail a large reorganization of the molecules. The crystal habits of the polymorphic forms can be used to distinguish them using microscopy. The $\alpha$ form typically crystallizes in elongated blocks with a high aspect ratio or needles, the $\beta$ and $\gamma$ forms in elongated blocks, and the $\delta$ form in plates.

DMU (Figure 1b) has two known polymorphic forms, which are enantiotropically related; form I is stable at high temperatures and form II at low temperatures. ${ }^{23}$ The thermodynamic transition temperature lies around $298 \mathrm{~K}$ in the presence of water, even though it is observed in the DSC at $324 \mathrm{~K}$, and at low relative humidity $(\mathrm{RH})$ it increases to 331 $\mathrm{K}^{24} \mathrm{DMU}$ is commercialized in form I. Form II of DMU transforms to form I at room temperature, but form II was not produced during these experiments. Furthermore, DMU is strongly hygroscopic, and it has a deliquescence point of $63.5 \%$ $\mathrm{RH}$ at $291 \mathrm{~K}^{25}$

As mentioned earlier, the influence of the addition of excipients on the phase transformation behavior of pyrazinamide has been studied by Baaklini et al. ${ }^{16}$ This study revealed that cospray drying of PZA and 1,3-dimethylurea (DMU) results in a mixture of the $\gamma$ form of PZA and form I of DMU, and this combination extends the lifetime of the high temperature form of PZA. Even after three years, the $\gamma$ form of PZA remains unchanged when it is spray dried with DMU, while it transforms within 1 week without DMU. The minimal amount of DMU required in the starting mixture to increase the lifetime of $\gamma$-PZA is about 5 mass $\%$, as was determined by Baaklini et al. ${ }^{16}$ Powder X-ray diffraction (PXRD) showed no sign of the formation of a cocrystal, whereas the binary phase diagram and Tammann plot of $\gamma$-PZA and DMU-I revealed no indication of a partial solid solution ${ }^{26,27}$ at the extremes of the phase diagram. ${ }^{24}$ The binary phase diagram and Tammann plot of $\gamma$-PZA with DMU-I are also included as Supporting Information for this work. Moreover, there was no shift in the PXRD patterns at high $\theta$ values, which also reduces the possibility of a solid solution. ${ }^{24}$ Furthermore, Raman mapping showed a homogeneous distribution of particles of about $10 \mu \mathrm{m}$ in size. ${ }^{16}$

The aim of this work is to understand the nature of the phase transition mechanism of $\gamma$-PZA to the low temperature forms. The influence of DMU on the kinetics of this process is investigated for varying conditions of relative humidity and crystallinity. Spray drying and milling, which are typical techniques used for the production of pharmaceuticals, ${ }^{4}$ are used to generate crystallites of PZA that are small enough to transform on a reasonable time-scale, so that the phase transition process can be monitored.

\section{EXPERIMENTAL SECTION}

2.1. Materials. Pyrazinamide ( $\geq 97.5 \%)$ was purchased from Acros Organics and Sigma-Aldrich, and 1,3-dimethylurea (DMU) $(\geq 95 \%)$ was purchased from Alfa Aesar, and used without further purification. DMU was stored under low relative humidity conditions, to avoid water uptake. Single crystals of the room temperature forms of PZA were grown by solvent evaporation. The $\delta$ form (block-shape) was grown from a $7 \mathrm{mg} / \mathrm{mL} \mathrm{PZA} \mathrm{in} \mathrm{acetone} \mathrm{solution} \mathrm{at} 277 \mathrm{~K}$, and the $\alpha$ 
form (needle-shape) was grown from a $11 \mathrm{mg} / \mathrm{mL}$ PZA aqueous solution at $277 \mathrm{~K}$, or a $13 \mathrm{mg} / \mathrm{mL}$ PZA aqueous solution at $293 \mathrm{~K}$. The polymorphic form was checked using PXRD.

2.2. Spray Drying. Spray drying experiments were performed using a Büchi B-290 laboratory-scale mini spray dryer, with a $0.7 \mathrm{~mm}$ diameter nozzle, operating in cocurrent mode. Several batches of PZA were spray dried using different amounts of solvent. Typically, a total mass of $1 \mathrm{~g}$, consisting of only PZA or PZA and DMU in various compositions, was dissolved in a 50/50 v\% mixture of $\mathrm{H}_{2} \mathrm{O}$ and acetone, with a total volume of $160 \mathrm{~mL}$. The cospray-dried samples are indicated by their composition in mass; PZA-DMU 50-50, PZADMU 70-30, and PZA-DMU 90-10, with a 50, 30, and 10 mass \% DMU content, respectively. Several samples of each composition were produced. DMU was also spray dried separately from a 50/50 v\% mixture of $\mathrm{H}_{2} \mathrm{O}$ and acetone with a total volume of $40 \mathrm{~mL}$. All samples were spray dried at $373 \mathrm{~K}$ inlet temperature, with the $\mathrm{N}_{2}$ flow meter set at $40 \mathrm{~mm}$, the aspirator at $100 \%$, and the pump at $20 \%$. Directly after spray drying, the polymorphic composition of each sample was analyzed using PXRD.

2.3. Lifetime and Storage. The polymorphic content of several physical mixtures of (co)spray-dried PZA with and without DMU were monitored over time using PXRD. The cospray-dried samples had a 50-50 mass ratio of PZA/DMU. These samples were stored in desiccators at different controlled relative humidities (RH). The following salt solutions were used at $293 \mathrm{~K}$ to obtain a specific constant $\mathrm{RH}: \mathrm{P}_{2} \mathrm{O}_{5}(0.5 \% \mathrm{RH})$ under vacuum conditions, $\mathrm{NaCl}$ $(75.5 \% \mathrm{RH})$, and $\mathrm{K}_{2} \mathrm{SO}_{4}(97.6 \% \mathrm{RH})$. Some samples were stored under ambient $\mathrm{RH}$ conditions, but at various temperatures ranging from 277 to $323 \mathrm{~K}$.

2.4. Sublimation Growth. Sublimation growth of $\gamma$-PZA was carried out in a home-built cylindrical chamber at atmospheric pressure. The temperature of the heating chamber was $443 \mathrm{~K}$, while the cold finger on which single crystals were formed was at $423 \mathrm{~K}$. The temperatures were controlled using a Digital Controller RKC instrument HA900, connected to two Rössler Type K thermocouples probing the temperature in the heating chamber and cold finger, respectively. Two Delta Elektronika SM 7020-D power supplies were used to amplify the signal from the temperature controller for the heating elements. Typically, $25 \mathrm{mg}$ of $\alpha$-PZA powder was sublimed, and single crystals were grown in about $2.5 \mathrm{~h}$.

2.5. Ball Milling. Ball milling experiments were conducted on a Retsch Mixer Mill 400, operating at $30 \mathrm{~Hz}$ for 5 times $2 \mathrm{~min}$. Typically, samples consisted of about $40 \mathrm{mg}$ of $\gamma$-PZA single crystals obtained by sublimation growth, with (four samples) or without (four samples) 5 $\mathrm{mg}$ of DMU ( $\sim 10$ mass \%) in a $2.0 \mathrm{~mL}$ Eppendorf round-bottom tube. In between the five consecutive ball milling runs, each sample was taken out of the apparatus and shaken manually, to ensure homogeneous mixing. Four samples were prepared by separate ball milling of PZA and DMU, and subsequent mixing in a 90-10 mass ratio, equal to the coball-milled samples.

2.6. Powder X-ray Diffraction. PXRD measurements were performed on a Bruker D8 AXS Advance X-ray diffractometer using $\mathrm{Cu} \mathrm{K} \alpha$ radiation. The diffraction patterns were collected in the angular range $3-30^{\circ}$ by steps of $0.04^{\circ}$ and with a counting time of $0.3 \mathrm{~s}$ per step for spray-dried samples, and steps of $0.17^{\circ}$ with a counting time of $1 \mathrm{~s}$ per step for ball-milled samples. The characteristic powder diffraction peaks are expressed in ${ }^{\circ} 2 \theta$.

2.7. Thermal Stage Polarization Microscopy. PZA single crystals were studied under a nitrogen atmosphere in a Linkam LTS420 thermal stage. The thermal stage was coupled to a Nikon Eclipse LV100 polarization microscope, and the microscope images were recorded with a digital camera. The temperature range varied from 293 to $443 \mathrm{~K}$, using heating rates between 5 and $20 \mathrm{~K} / \mathrm{min}$.

2.8. Scanning Electron Microscopy. Scanning electron microscopy (SEM) images of spray-dried PZA samples were obtained with a JEOL JCM-5000 NeoScope instrument (secondary scattering electron) at an accelerated voltage of $10 \mathrm{kV}$. Powder samples were fixated on a SEM stub with carbon adhesive discs and coated with gold using a NeoCoater MP-19020NCTR sputter coater. SEM images of ball-milled PZA samples were obtained with a Phenom 800-03103-02 instrument, and the samples were coated with gold using a Cressington 108 auto Sputter Coater.

\section{RESULTS AND DISCUSSION}

3.1. Mechanism of Phase Transition. In order to understand the mechanism of the phase transition from the high temperature form $\gamma$-PZA to the low temperature forms, we first studied the phase transition in the reverse direction during heating. Single crystals of $\delta$-PZA and $\alpha$-PZA were grown from different solvents, acetone and water respectively, and have been monitored as a function of temperature using thermal stage polarization microscopy at various heating rates. Heating with $10 \mathrm{~K} / \mathrm{min}$ (Figure 3) shows that the $\delta \rightarrow \gamma$ phase

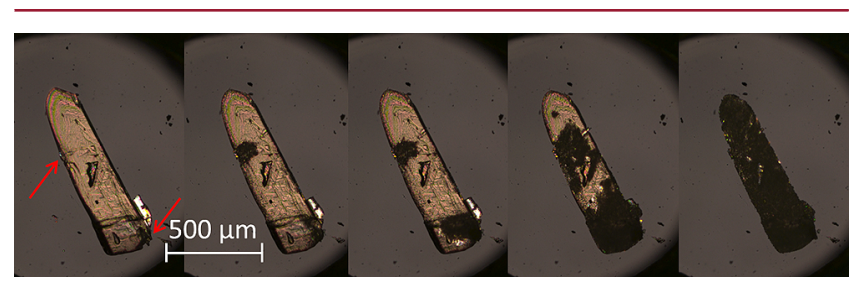

Figure 3. Thermal microscopy snapshots of the $\delta \rightarrow \gamma$ phase transition of PZA during heating at $10 \mathrm{~K} / \mathrm{min}$. The red arrows indicate defect sites where the phase transition starts.

transition at $407 \mathrm{~K}$ follows a defect-mediated nucleation-andgrowth mechanism, since the nucleation of the new phase occurs at visible defect sites. Moreover, the new phase spreads quite uniformly in all directions, thereby showing no orientational relationship between the two polymorphic forms. Furthermore, the spread is relatively slow, indicating that the energy barrier for propagation is relatively high for this transition, and the crystal completely loses its polarization color in this plane, due to deterioration of the crystal quality. During the final stage of the transition, around $423 \mathrm{~K}$, the crystal starts to evaporate due to the high vapor pressure at this temperature. The same holds for the $\alpha \rightarrow \gamma$ phase transition; only the observed transition temperature is higher.

The observed transition temperature increases with increasing heating rate, indicating kinetic hindrance of the solid-state phase transition, as was also observed by Castro et al. ${ }^{17}$ These thermal measurements show that the $\delta \rightarrow \gamma$ and $\alpha \rightarrow \gamma$ transitions are reconstructive, which implies that the reverse $\gamma$ $\rightarrow \delta / \alpha$ transitions are probably reconstructive as well. This is not surprising, since the phase transition toward or from the $\gamma$ form requires a large reorganization of the molecules, due to the absence of head-to-head dimers in the $\gamma$ form.

The morphology and phase transition mechanism of spraydried samples of PZA was studied using SEM, as is shown in Figure 4. Directly after spray drying, a $\gamma$-PZA powder sample (Figure 4a) consists of small elongated block-shaped crystallites with a length of $<10 \mu \mathrm{m}$. After storage for 5 days at ambient conditions, the powder had partially transformed to the $\alpha$ form, as was determined using PXRD. The SEM image (Figure $4 \mathrm{~b}$ ) shows a mixture of a few large faceted elongated blocks of 20$50 \mu \mathrm{m}$ in length and clusters of small crystallites of $<10 \mu \mathrm{m}$. Other spray-dried PZA powders, that were stored for longer periods and measured after storage, showed various morphologies ranging from elongated blocks of $100 \mu \mathrm{m}$ in length for $\alpha$ PZA (Figure 4c) to thin plates of about $20 \mu \mathrm{m}$ in size for $\delta$ PZA (Figure 4d).

It appears from these images that in general the final crystal phase has larger crystals than the spray-dried $\gamma$-PZA. This is 


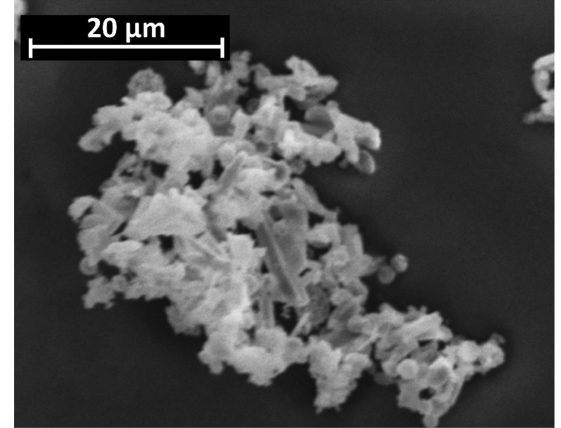

(a)

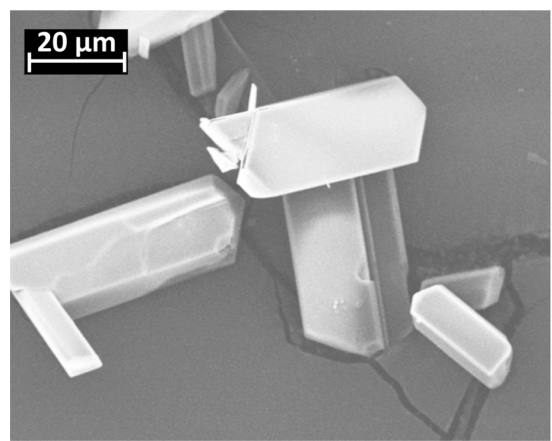

(c)

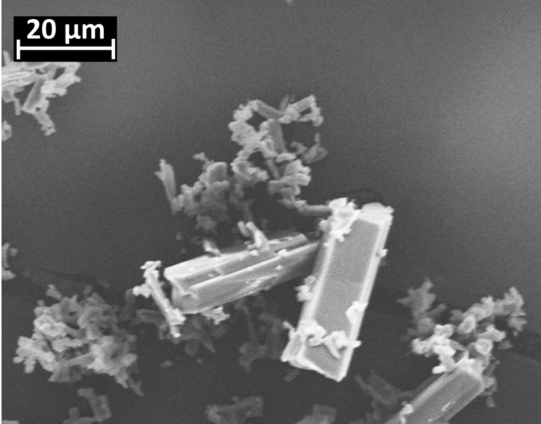

(b)

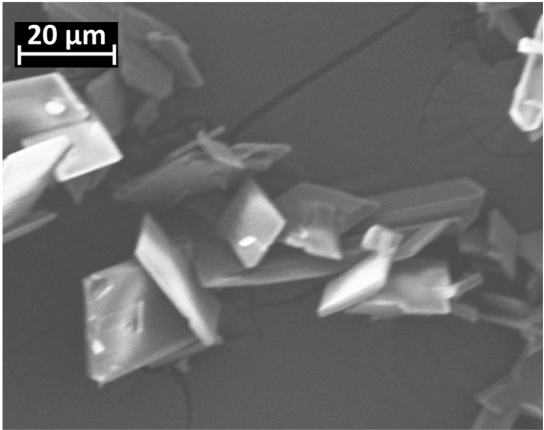

(d)

Figure 4. SEM images of a spray-dried PZA sample (a) directly after spray drying ( $\gamma$ form), (b) after 5 days of storage under ambient conditions $(\gamma$ and $\alpha$ forms); other spray-dried PZA samples stored for (c) 23 days ( $\alpha$ form), and (d) 49 days ( $\delta$ form). Note the larger magnification in (a).

remarkable, since in most solid-state phase transitions the mother crystal breaks down into smaller daughter crystals or remains more or less intact. ${ }^{28}$ If the daughter crystals are indeed larger, this is probably the result of a recrystallization process via the vapor phase. ${ }^{29}$ Pyrazinamide has a relatively high vapor pressure at elevated temperatures and can therefore be recrystallized to the high temperature $\gamma$ polymorph by sublimation growth. ${ }^{30}$ Apparently, the vapor pressure of PZA is also sufficiently high at room temperature for a vapormediated phase transition mechanism. Most vapor-mediated phase transitions described in the literature entail transformations from an anhydrate to a hydrate or solvate form through the aid of solvent vapor. ${ }^{31-33}$ In case the solvent vapor does not cause but merely catalyzes the transformation, the term solvent-catalyzed transformation is used. This involves a direct solid-solid transformation accelerated by the catalytic effect of solvent in the liquid or vapor state. ${ }^{7}$ To the best of our knowledge, few studies have been done on vapor-mediated phase transitions in which the compound itself evaporates and recrystallizes to another polymorphic form within a reasonable time span. ${ }^{29}$

3.2. Effect of Relative Humidity. As mentioned before, the phase transition kinetics of $\gamma$-PZA to the other low temperature polymorphs is delayed by the addition of DMU. A possible mechanism of stabilization is the attraction of water by $\mathrm{DMU}$, if water plays a role in the phase transition of $\gamma$-PZA. Since DMU shows deliquescence above $63.5 \% \mathrm{RH}$, the effect of DMU is expected to be influenced by the relative humidity, due to the strong affinity between DMU and water. Therefore, we investigated the influence of relative humidity on the stability of spray-dried pure $\gamma$-PZA and $\gamma$-PZA with DMU-I in further detail.
3.2.1. $\gamma$-PZA. The transition rate of $\gamma$-PZA to the other low temperature polymorphs was monitored as a function of $\mathrm{RH}$ and temperature using ex situ PXRD. First, the lifetime of $\gamma$ PZA — without DMU - after spray drying was measured using PXRD after storage at various RH values. Figure 5 shows that

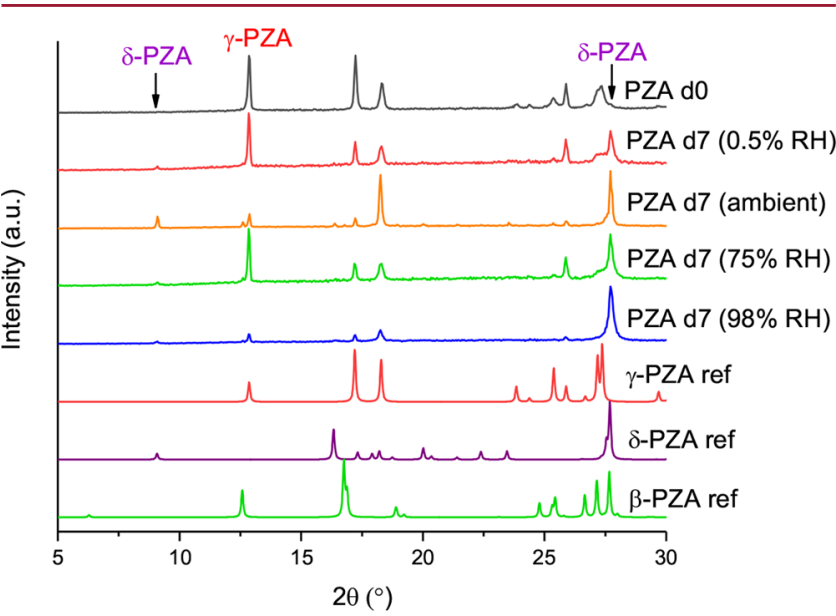

Figure 5. PXRD patterns of PZA after spray drying (day 0) and after storage for 7 days at various relative humidities. After storage, $\gamma$-PZA transforms to the $\delta$ form in this case.

directly after spray drying, PZA crystallizes only in the $\gamma$ form. After storage for 7 days at a specific $\mathrm{RH}$ ranging from 0.5 to 97.6\%, PZA partially transforms to the $\delta$ form. The transformation rate to the $\delta$ form increases with higher $\mathrm{RH}$, as can be clearly observed from the difference in intensity of the typical $\delta$-PZA peak at $27.8^{\circ}$. In general, the obtained PZA 
polymorphic form after transition of the various samples investigated varies and is not clearly related to the $\mathrm{RH}$; the $\delta, \beta$, and $\alpha$ forms have all been observed and often in a mixture. Since pure PZA showed no significant mass gain as a function of $\mathrm{RH}$ in dynamic vapor sorption (DVS) experiments, it is unlikely that the phase transition mechanism is solventmediated, which would involve deliquescence and subsequent recrystallization of another polymorphic form. However, the polymorphic transformation rate could be slightly enhanced by water vapor through a solvent vapor-catalyzed transformation, which entails that a monolayer of water molecules from the vapor is adsorbed and increases the mobility at the surface. In conclusion, even at the lowest $\mathrm{RH}$ values the phase transition to a low temperature form starts already within a week.

3.2.2. $\gamma-P Z A$ and DMU-I. In order to further study the effect of DMU on the lifetime of $\gamma$-PZA, PZA was also cospray dried with DMU in a 50:50 mass-ratio (PZA-DMU 50-50) and stored under various $\mathrm{RH}$ conditions. The lifetime-extending effect of DMU is expected to disappear above its deliquescence point. As is shown in Figure 6, PZA crystallizes in the $\gamma$ form

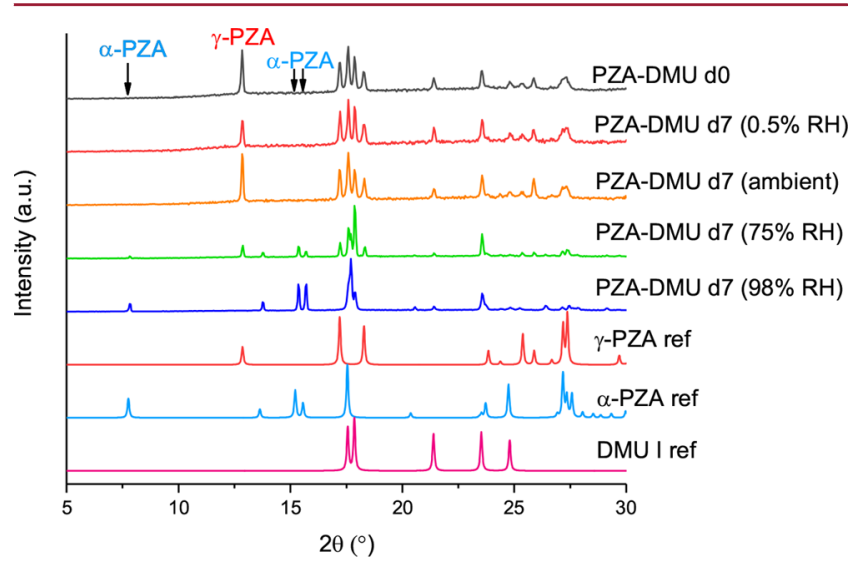

Figure 6. PXRD patterns of PZA-DMU after spray drying (day 0) and after storage for 5-7 days under different $\mathrm{RH}$. During storage at ambient conditions or low relative humidity, no phase transition is observed.

and DMU in form I during spray drying. After storage under high relative humidity conditions (>75\%) for 5-7 days, $\gamma$-PZA indeed partially transforms to the $\alpha$ form. Again, as was observed for pure $\gamma$-PZA, the transformation rate increases with higher relative humidity, as can be seen from the difference in intensity of the typical $\alpha$ form peaks at 7.8, 13.6, 15.2, and $15.6^{\circ}$. Above the deliquescence point of DMU, the transformation rate of $\gamma$-PZA with DMU is higher than without DMU. These PZA-DMU samples were clearly more wet due to water uptake by DMU than the pure PZA samples, probably leading to a solvent-mediated phase transition. Storage under ambient conditions or at low relative humidity did not induce a transition of $\gamma$-PZA-DMU, during a storage period of two months. Another PZA-DMU sample described earlier by some of the authors ${ }^{16}$ was stored for more than three years under ambient conditions in a closed transparent vial without transforming.

From this we confirm that DMU extends the lifetime of the high temperature $\gamma$ form of PZA for cospray-dried samples. At a high $\mathrm{RH}$, the polymorphic transformation of $\gamma$-PZA is not inhibited due to the hygroscopic character of DMU, which quickly becomes deliquescent under these conditions. DMU acts as a polymorphic transition retardant of $\gamma$-PZA, but only in the solid state and below $75 \% \mathrm{RH}$ at RT.

3.3. Interaction between $\gamma$-PZA and DMU-I. To further look into the nature of the interaction between $\gamma$-PZA and DMU-I we will distinguish between three cases: cospray-dried samples where DMU and PZA were mixed during spray drying, i.e., during the nucleation-and-growth phase, and two cases of sublimation-grown $\gamma$-PZA for which the relatively large crystals are ball milled to reduce the crystal size to sizes more comparable to the spray-dried samples. In one case of ball milling, $\gamma$-PZA and DMU-I are ball milled together, which is a highly energetic event. In the other case, $\gamma$-PZA and DMU-I are ball milled separately and then physically mixed, which allows for less interaction between PZA and DMU. Therefore, the amount of interaction between $\gamma$-PZA and DMU-I decreases from spray-dried to coball-milled to physically mixed.

The spray-dried PZA-DMU samples with different compositions were measured using SEM after storage for several weeks under ambient conditions. They showed that the morphology appears to change with composition, from faceted elongated blocks or plates for PZA-DMU 90-10 (Figure 7a) to a mixture of small (DMU) and large $(\gamma$-PZA) crystals for PZADMU 70-30 (Figure 7b) to irregularly shaped crystals for PZA-DMU 50-50 (Figure 7c). All samples were confirmed with PXRD to consist of only $\gamma$-PZA and DMU-I. There was no notable difference in the SEM images between samples measured directly after spray drying and after storage for several weeks. No large PZA crystals of low temperature forms appear in the presence of DMU. We believe the phase transition inhibition by DMU is caused either by inhibiting the nucleation of low temperature polymorphic forms of PZA, or by inhibiting the evaporation of $\gamma$-PZA. To exclude the possible influence of growing crystals from a solution of both compounds, which is the case for spray drying, $\gamma$-PZA and DMU-I were separately crystallized and subsequently ball milled, either together or independently.

Sublimation growth of PZA typically yielded single crystals of $\gamma$-PZA of typically $300-500 \mu \mathrm{m}$ in size. Several single crystals

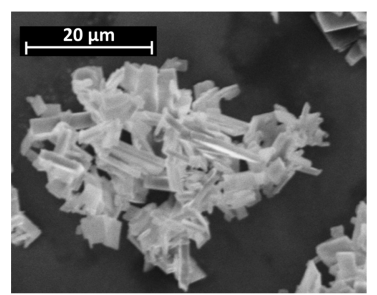

(a)

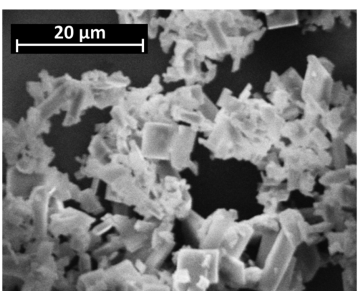

(b)

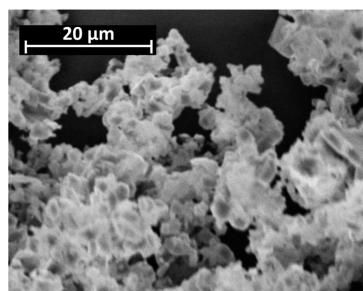

(c)

Figure 7. SEM images of three spray-dried PZA-DMU samples stored for the period of time in between brackets with the following compositions: (a) 90-10 (15 days), (b) 70-30 (9 days), (c) 50-50 (42 days). 
produced using a home-built sublimation setup were measured after 15 months of storage under ambient conditions using SCXRD. Unit cell determinations at room temperature showed these crystals were still the $\gamma$ form of PZA, implying that single crystals grown by sublimation have a much longer lifetime of the $\gamma$ form than spray-dried powders, due to the crystallite size and lack of defects. After sublimation growth, the single crystals were ground to smaller crystallites using manual grinding or ball milling to speed up the transformation to the low temperature forms of PZA. However, reducing the size of pure $\gamma$-PZA crystals to $50-300 \mu \mathrm{m}$ by means of manual grinding does not accelerate the phase transition to the low temperature polymorphs sufficiently, since no transformation was observed within three months. Therefore, the effect of DMU on inhibiting the already slow phase transition of PZA was not investigated for these crystals.

High-energy ball milling can induce the $\gamma \rightarrow \alpha$ phase transition of PZA, as was shown by Cherukuvada et al. ${ }^{18} \mathrm{We}$ optimized our ball milling procedure to achieve a phase transition within a few days after the ball milling. The procedure entailed five times 2 min of milling at $30 \mathrm{~Hz}$ with 1 min breaks in between, during which the samples were shaken manually for homogeneity. This procedure resulted in $\gamma$-PZA crystallites with a size of approximately $50 \mathrm{~nm}$, as was determined from PXRD powder patterns using Rietveld refinement, which start to transform to $\alpha$-PZA within 2 days. Directly after ball milling, PXRD indicates a significant amount of amorphous material, see Figure 8. The $\gamma \rightarrow \alpha$ transformation

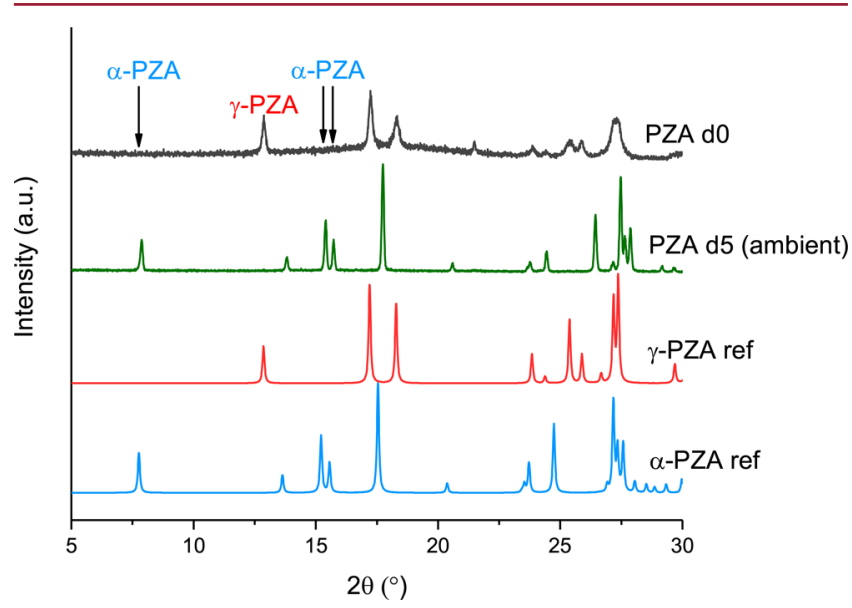

Figure 8. PXRD of ball-milled $\gamma$-PZA sublimation growth crystals, directly after ball milling and after 5 days of storage.

was almost complete after 5 days. The powder patterns of PZA directly after ball-milling compared to the pattern after 5 days, shown in Figure 8, reveal that the peaks become narrower and the background is lower. This indicates the crystallite size increases to $10 \mu \mathrm{m}$ and the sample becomes less amorphous. This is visualized in the SEM images in Figure 9a directly after ball milling, where many small crystallites are clustered together in a large particle, and Figure 9b after 4 days, where well-faceted crystallites of $\alpha$-PZA are formed. The shape and relatively large size of the new crystals indicate a relatively slow and controlled recrystallization process took place, similar to spray-dried PZA. Thus again a vapor-mediated phase transition appears the most likely mechanism, and the results of the ball-milled samples and the spray-dried samples can be directly compared. Amorphous material has a higher vapor pressure than the corresponding

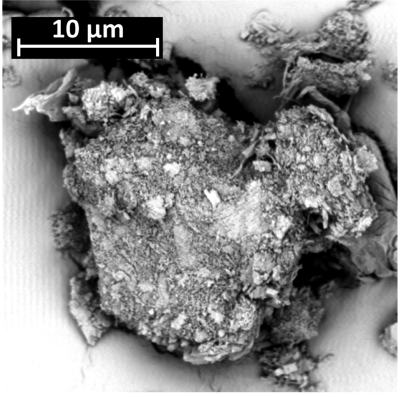

(a)

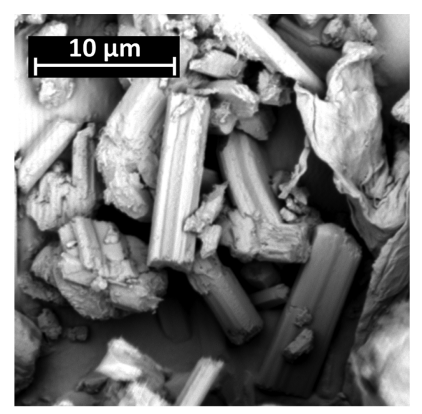

(b)
Figure 9. SEM images of ball-milled $\gamma$-PZA sublimation growth crystals, (a) directly after ball milling small crystallites of $50 \mathrm{~nm}$ are clustered together, and (b) after 4 days $\alpha$-PZA crystals of $10 \mu \mathrm{m}$ are formed.

crystallized material. This, combined with the relatively small size and high defect density of the high-energy ball-milled samples, will lead to an increase in the transition rate for the vapor-mediated mechanism, and possibly also solid-solid phase transitions for the defected crystallites.

To study the effect of DMU for the ball-milled samples, either before or after ball milling 10 mass \% of DMU was added to some samples, to see whether the interaction during the coball-milling is essential for the lifetime-extending effect or that only physical mixing of separately ball-milled PZA and DMU is enough. Co-ball milling of PZA and DMU resulted in a lifetime of $\gamma$-PZA of 1-2 months, but after that the samples transformed to $\alpha$ and/or $\delta$-PZA. Separate ball milling and subsequent physical mixing by manual shaking resulted only in temporary lifetime extension of several days to 2 weeks. Thus, ball-milling with DMU is much less effective in extending the lifetime of the high temperature form of PZA than cospray drying with DMU. Moreover, DMU influences the morphology of PZA in spray drying experiments; more DMU results in smaller, less well-faceted crystallites, as was shown in Figure 7. Therefore, we conclude that there is a surface interaction between PZA and DMU that hampers the growth of $\gamma$-PZA during spray drying and also slows down the kinetics of the $\gamma$ PZA phase transition to the low temperature forms. This interaction is decreasingly effective for delaying the phase transition in cospray drying, coball milling, and separate ball milling. This surface interaction probably reduces the vapor pressure of $\gamma$-PZA such that the vapor-mediated phase transition is delayed or even inhibited. Another possibility is inhibition of the nucleation of the other polymorphic forms, by blocking the formation of PZA dimers, which are the building blocks of those three forms of PZA. In order to determine the nature of the lifetime-extending effect, the interaction between PZA and DMU should be studied on a molecular scale, e.g., using computational modeling and surface techniques.

\section{CONCLUSION}

The pharmaceutical compound pyrazinamide (PZA) has high energy barriers for the solid-state phase transitions between the high temperature $\gamma$ form and the three low temperature polymorphic forms, and vice versa. This is due to the difference between the orientation of one out of two neighboring molecules in head-to-tail chains vs head-to-head dimers, respectively. Therefore, the phase transition is kinetically hindered in both directions. This work shows that the vapor pressure of the high temperature $\gamma$ polymorph of PZA is high 
enough for a vapor-mediated phase transition mechanism to be dominant at room temperature for the $\gamma \rightarrow \alpha / \beta / \delta$ transition in spray-dried and ball-milled samples with a large surface area. For the reverse $\alpha / \beta / \delta \rightarrow \gamma$ phase transformation, which occurs at relatively high temperatures, a nucleation-and-growth solidsolid phase transition mechanism is dominant. This mechanism is orders of magnitude faster than the vapor-mediated mechanism. Possibly, the vapor pressure of the $\gamma$-form is higher than that of the other forms, due to the lack of dimers in the crystal structure of $\gamma$-PZA. The lifetime of the high temperature $\gamma$-form of PZA can be greatly extended up to several years at room temperature by the addition of DMU during spray drying. Co-spray drying of PZA with DMU is more effective in extending the lifetime of $\gamma$-PZA than (co)ball milling, probably due to a better mixing of the two compounds. For relative humidity values above the deliquescence point of $\mathrm{DMU}$, its lifetime-extending effect on $\gamma$-PZA disappears. The inhibiting effect of DMU seems very specific, and all the other urea derivatives tested so far do not have this property. Although it is not possible at this stage of the study to rule out that there might be some minor degree of incorporation of DMU, we believe that the surface interaction between PZA and DMU slows down the kinetics of the vapor-mediated $\gamma$-PZA phase transition, most likely by preventing the evaporation of $\gamma$ PZA. Alternatively, DMU might prevent the nucleation of the low temperature forms of PZA.

\section{ASSOCIATED CONTENT}

\section{S Supporting Information}

The Supporting Information is available free of charge on the ACS Publications website at DOI: 10.1021/acs.cgd.7b01550.

Schematic metastable binary phase diagram of $\gamma$-PZA and DMU-I (Figure 1) (PDF)

\section{AUTHOR INFORMATION}

\section{Corresponding Authors}

*(H.M.C.) E-mail: h.cuppen@science.ru.nl.

*(G.C.) E-mail: gerard.coquerel@univ-rouen.fr. ORCID ${ }^{\circledR}$

M. M. H. Smets: 0000-0003-1938-2099

Notes

The authors declare no competing financial interest.

\section{ACKNOWLEDGMENTS}

The authors are grateful for financial support from the VIDI research program 700.10 .427 financed by the Netherlands Organisation for Scientific Research (NWO) and the ERC grant from the European Research Council (ERC-2010-StG, Grant Agreement 259510-KISMOL).

\section{REFERENCES}

(1) Bernstein, J. Polymorphism in Molecular Crystals; Oxford Science Publications, 2002.

(2) Chemburkar, S. R; et al. Dealing with the Impact of Ritonavir Polymorphs on the Late Stages of Bulk Drug Process Development. Org. Process Res. Dev. 2000, 4, 413-417.

(3) Lee, E. H. A practical guide to pharmaceutical polymorph screening \& selection. Asian J. Pharm. Sci. 2014, 9, 163-175.

(4) Zhou, D. Understanding Physicochemical Properties for Pharmaceutical Product Development and Manufacturing II: Physical and Chemical Stability and Excipient Compatibility. J. Valid. Technol. 2009, 15, 36-47.
(5) Singhal, D.; Curatolo, W. Drug polymorphism and dosage form design: a practical perspective. Adv. Drug Delivery Rev. 2004, 56, 335347.

(6) Blagden, N.; de Matas, M.; Gavan, P.; York, P. Crystal engineering of active pharmaceutical ingredients to improve solubility and dissolution rates. Adv. Drug Delivery Rev. 2007, 59, 617-630.

(7) Aucamp, M. E.; Liebenberg, W.; Stieger, N. In Advanced Topics in Crystallization; Mastai, Y., Ed.; InTech, 2015; Chapter 1.

(8) Llinàs, A.; Goodman, J. M. Polymorph control: past, present and future. Drug Discovery Today 2008, 13, 198-210.

(9) Lee, E. H.; Byrn, S. R. Stabilization of Metastable Flufenamic Acid by Inclusion of Mefenamic Acid: Solid Solution or Epilayer? J. Pharm. Sci. 2010, 99, 4013-4022.

(10) Song, R.-Q; Colfen, H. Additive controlled crystallization. CrystEngComm 2011, 13, 1249-1276.

(11) Padrela, L.; Zeglinski, J.; Ryan, K. M. Insight into the Role of Additives in Controlling Polymorphic Outcome: $\mathrm{A} \mathrm{CO}_{2}$-Antisolvent Crystallization Process of Carbamazepine. Cryst. Growth Des. 2017, 17, 4544-4553.

(12) Simone, E.; Steele, G.; Nagy, Z. K. Tailoring crystal shape and polymorphism using combinations of solvents and a structurally related additive. CrystEngComm 2015, 17, 9370-9379.

(13) Simone, E.; Cenzato, M.; Nagy, Z. A study on the effect of the polymeric additive HPMC on morphology and polymorphism of ortho-aminobenzoic acid crystals. J. Cryst. Growth 2016, 446, 50-59.

(14) Kamali, N.; Erxleben, A.; McArdle, P. Unexpected Effects of Catalytic Amounts of Additives on Crystallization from the Gas Phase: Depression of the Sublimation Temperature and Polymorph Control. Cryst. Growth Des. 2016, 16, 2492-2495.

(15) Ewen, B.; Strobl, G. R.; Richter, D. Phase transitions in crystals of chain molecules. Relation between defect structures and molecular motion in the four modifications of $\mathrm{n}-\mathrm{C}_{33} \mathrm{H}_{68}$. Faraday Discuss. Chem. Soc. 1980, 69, 19-31.

(16) Baaklini, G.; Dupray, V.; Coquerel, G. Inhibition of the spontaneous polymorphic transition of pyrazinamide $\gamma$ form at room temperature by co-spray drying with 1,3-dimethylurea. Int. J. Pharm. 2015, 479, 163-170.

(17) Castro, R. A. E.; Maria, T. M. R.; Évora, A. O. L.; Feiteira, J. C.; Silva, M. R.; Beja, A. M.; Canotilho, J. a.; Eusébio, M. E. S. A New Insight into Pyrazinamide Polymorphic Forms and their Thermodynamic Relationships. Cryst. Growth Des. 2010, 10, 274-282.

(18) Cherukuvada, S.; Thakuria, R.; Nangia, A. Pyrazinamide Polymorphs: Relative Stability and Vibrational Spectroscopy. Cryst. Growth Des. 2010, 10, 3931-3941.

(19) Bernstein, J.; Davey, R.; Henck, J. Concomitant Polymorphs. Angew. Chem., Int. Ed. 1999, 38, 3440-3461.

(20) Nangia, A.; Srinivasulu, A. CSD Communication.

(21) Rajalakshmi, G.; Hathwar, V. R.; Kumaradhas, P. Intermolecular interactions, charge-density distribution and the electrostatic properties of pyrazinamide anti-TB drug molecule: an experimental and theoretical charge-density study. Acta Crystallogr., Sect. B: Struct. Sci., Cryst. Eng. Mater. 2014, 70, 568-579.

(22) Jarzembska, K. N.; Hoser, A. A.; Kamiński, R.; Madsen, A. O.; Durka, K.; Woźniak, K. Combined Experimental and Computational Studies of Pyrazinamide and Nicotinamide in the Context of Crystal Engineering and Thermodynamics. Cryst. Growth Des. 2014, 14, $3453-3465$

(23) Näther, C.; Döring, C.; Jess, I.; Jones, P. G.; Taouss, C. Thermodynamic and structural relationships between the two polymorphs of 1,3-dimethylurea. Acta Crystallogr., Sect. B: Struct. Sci., Cryst. Eng. Mater. 2013, 69, 70-76.

(24) Baaklini, G. The impacts of Spray Drying on the polymorphism of pharmaceutical and organic compounds. Ph.D. thesis, Universite de Rouen, https://tel.archives-ouvertes.fr/tel-01685045, 2015.

(25) Clow, A. Deliquescence in Urea and Methyl-ureas. Nature 1940, 146, 26.

(26) Coquerel, G. Thermodynamic Predictions of Physical Properties - Prediction of Solid Solutions in Molecular Solutes Exhibiting Polymorphism. Chem. Eng. Technol. 2006, 29, 182-186. 
(27) Costa, M. C.; Sardo, M.; Rolemberg, M. P.; Coutinho, J. A. P.; Meirelles, A. J.; Ribeiro-Claro, P.; Krähenbühl, M. The solid-liquid phase diagrams of binary mixtures of consecutive, even saturated fatty acids. Chem. Phys. Lipids 2009, 160, 85-97.

(28) Mnyukh, Y. Fundamentals of Solid-State Phase Transitions, Ferromagnetism and Ferroelectricity, 2nd ed.; Direct Scientific Press, 2010.

(29) Jiang, S.; Jansens, P. J.; ter Horst, J. H. Mechanism and Kinetics of the Polymorphic Transformation of o-Aminobenzoic Acid. Cryst. Growth Des. 2010, 10, 2123-2128.

(30) Blokhina, S.; Sharapova, A.; Ol'khovich, M.; Volkova, T.; Perlovich, G. Vapor pressures and thermodynamic sublimation of antitubercular drugs. J. Therm. Anal. Calorim. 2015, 120, 1053-1060. (31) Chakravarty, P.; Govindarajan, R.; Suryanarayanan, R. Investigation of Solution and Vapor Phase Mediated Phase Transformation in Thiamine Hydrochloride. J. Pharm. Sci. 2010, 99, 39413952.

(32) Goodfellow, B. W.; Korgel, B. A. Reversible Solvent VaporMediated Phase Changes in Nanocrystal Superlattices. ACS Nano 2011, 5, 2419-2424.

(33) Davies, K.; Bourne, S. A.; Oliver, C. L. Solvent- and VaporMediated Solid-State Transformations in 1,3,5-Benzenetricarboxylate Metal-Organic Frameworks. Cryst. Growth Des. 2012, 12, 1999-2003. 